\title{
Estimation of the Shear Stress Parameter of a Power-Law Fluid
}

\author{
Samer S. Al-Ashhab and Rubayyi T. Alqahtani \\ Department of Mathematics and Statistics, Al Imam Mohammad Ibn Saud Islamic University, \\ P.O. Box 90950, Riyadh 11623, Saudi Arabia
}

Correspondence should be addressed to Samer S. Al-Ashhab; ssashhab@imamu.edu.sa

Received 28 May 2016; Accepted 27 June 2016

Academic Editor: Mohamed Abd El Aziz

Copyright (C) 2016 S. S. Al-Ashhab and R. T. Alqahtani. This is an open access article distributed under the Creative Commons Attribution License, which permits unrestricted use, distribution, and reproduction in any medium, provided the original work is properly cited.

We apply the Adomian decomposition method to a power-law problem for solutions that do not change the sign of curvature. In particular we consider solutions with positive curvature. The power series obtained via the Adomian decomposition method is used to estimate the shear stress parameter as well as the instant of time where the solution reaches its terminal point of a steady state. We compare our results with estimates obtained via numerical integrators. More importantly we illustrate that the error is predictable and can be reduced without further effort or using higher order terms in the approximating series.

\section{Introduction}

Due to its interesting mathematical properties and huge number of applications, the problem of non-Newtonian fluid flow has attracted attention from a large number of researchers. The most commonly used model for non-Newtonian fluid mechanics is the Ostwald-de Waele model with a power-law rheology. The value of the power-law index $n=1$ corresponds to a Newtonian fluid, while $n>1$ describes a dilatant or shearthickening fluid and $0<n<1$ describes a pseudo-plastic or shear-thinning fluid. The corresponding nonlinear thirdorder problem is the following:

$$
\left(\left|f^{\prime \prime}\right|^{n-1} f^{\prime \prime}\right)^{\prime}+\frac{1}{n+1} f f^{\prime \prime}=0
$$

subject to

$$
\begin{aligned}
& f(0)=0, \\
& f^{\prime}(0)=\epsilon, \\
& f^{\prime}(\eta) \longrightarrow 1 \quad \text { as } \eta \longrightarrow \infty
\end{aligned}
$$

where the primes are derivatives with respect to $\eta$. To simplify notation let $y=f, x=\eta$, where these are referred to as the similarity variables of the problem. Equation (1) can be written, for positive curvature solutions $y^{\prime \prime}>0$, as

$$
y^{\prime \prime \prime}=\beta y\left(y^{\prime \prime}\right)^{2-n} ; \quad \beta=\frac{-1}{n(n+1)},
$$

where solution domains with $y^{\prime \prime}=0$ are ruled out for $n>2$. (Those domains are naturally ruled out since the problem is solved until the solution reaches its terminal state $y^{\prime \prime}=0$ at, say, $x=t_{f}$.)

Earlier studies of this problem date back to the work by Blasius [1] where he considered the case where $n=1$. Existence and uniqueness of similar power-law problems have also been studied by Guedda and Hammouch [2, 3], Nachman and Taliaferro [4], and Zheng et al. [5] to mention a few. In particular, in [5] the authors studied the problem with identical boundary conditions to the ones considered here but with coefficient equal to 1 on the second term involving $f f^{\prime \prime}$ in (1) above. Their approach, however, enabled them to obtain bounds on an important parameter, the skin friction coefficient, in addition to proving existence and uniqueness. They compared their results to numerical estimates to illustrate their accuracy and it was observed that their bounds (upper and lower for skin friction) were very close for large $n$ so that an accurate estimate can readily be obtained by taking, for example, the average of those two bounds. We will not be able to compare our results to 
theirs directly, however, due to the coefficient on the second term in (1) above which somewhat changes the "dynamics" of the governing equation and its solutions (even though the deviation may be small for some smaller values of $n$ ). Howell et al. [6] studied the problem in the context of momentum and heat transfer. In [7] the authors, Chen et al., considered boundary layer flow of an electrically conducting non-Newtonian fluid in the presence of a magnetic field. Their setting required two additional terms in the equation involving $f^{\prime}$ and $\left(f^{\prime}\right)^{2}$ explicitly, but with slightly different boundary conditions. They proceeded to utilize Crocco variables and the Adomian decomposition method (ADM) to obtain approximate solutions to the problem (in the Crocco variables domain) as well as estimate the value of the skin friction coefficient and they illustrated the accuracy of their findings. Further numerical and approximate solutions can be found in [8-11], for example.

Existence and uniqueness were established for the exact current problem at hand in [12] where all possible values of the power-law index $n$ were considered and it was assumed that $f^{\prime \prime} \geq 0$ (or $y^{\prime \prime} \geq 0$ ). In $[13,14]$ one finds extensive discussion of the general theory of non-Newtonian boundary layer flow fluids including the physical derivation of the power-law problem. Lastly we note that in [15] a unified equation governing positive and negative curvatures for non-Newtonian power-law fluids was derived and analytical solutions were obtained.

In Section 2 we derive the first few terms in a power series expansion (using the ADM) for the solution to the problem and use those terms to approximate the solution where our approach will help in obtaining estimates not only for the shear stress parameter but also for the instant of time $t_{f}$, where the solution virtually reaches its terminal point, where $y^{\prime}\left(t_{f}\right)=1, y^{\prime \prime}\left(t_{f}\right)=0$, within $10^{-4}$. In Section 3 we compare our results to numerical estimates obtained via numerical computer integrators.

\section{The Series Solution}

To find the power series solution to our problem we utilize the Adomian decomposition method: observe that the solution $y$ satisfies

$$
y=\epsilon x+\alpha x^{2}+L^{-1}(F)
$$

where the condition $y^{\prime \prime}(0)=2 \alpha$ replaces the condition at infinity and where $F=\beta y\left(y^{\prime \prime}\right)^{2-n}$ from (3) above. $L^{-1}=$ $\int_{0}^{x} \int_{0}^{x} \int_{0}^{x}(\cdot) d x d x d x$ is an operator, where $L=d^{3} / d x^{3}$ is the operator applied to $y$ on the left hand side of (3) as $L[y]$. Now expand $y$ in an infinite series of the form $y=\sum_{i=0}^{\infty} y_{i}$. The decomposition method is based on substituting this series for $y$ into (4) above and extracting the terms out one by one by a linearization process of the operator. In particular, $y_{i}$ 's are obtained via

$$
y_{i}=L^{-1}\left(A_{i-1}\right), \quad i=1,2,3, \ldots,
$$

where the $A_{i}$ 's are referred to as the Adomian polynomials and are given by

$$
\begin{aligned}
A_{0}= & F\left(y_{0}\right) ; \\
A_{1}= & \frac{\partial F}{\partial f}\left(y_{0}\right) \cdot y_{1}+\frac{\partial F}{\partial f^{\prime \prime}}\left(y_{0}\right) \cdot y_{1}^{\prime \prime} ; \\
A_{2}= & \frac{\partial F}{\partial f}\left(y_{0}\right) \cdot y_{2}+\frac{\partial F}{\partial f^{\prime \prime}}\left(y_{0}\right) \cdot y_{2}^{\prime \prime}+\frac{\partial^{2} F}{\partial f \partial f^{\prime \prime}}\left(y_{0}\right) \\
& \cdot y_{1} y_{1}^{\prime \prime}+\frac{\partial^{2} F}{\partial^{2} f^{\prime \prime}}\left(y_{0}\right) \cdot \frac{\left(y_{1}^{\prime \prime}\right)^{2}}{2}
\end{aligned}
$$

and where we have skipped writing $\left(\partial^{2} F / \partial^{2} f\right)\left(y_{0}\right) \cdot\left(\left(y_{1}\right)^{2} /\right.$ $2)=0$ from $A_{2}$. This in turn yields the explicit expressions (with $y_{0}=\epsilon x+\alpha x^{2}$ and using $\gamma=2 \alpha$ ):

$$
\begin{aligned}
A_{0}= & \beta\left(\epsilon x+\alpha x^{2}\right)(\gamma)^{2-n}, \\
A_{1}= & \beta(2 \alpha)^{2-n} y_{1}+\beta(2-n)\left(\epsilon x+\alpha x^{2}\right)(\gamma)^{1-n} y_{1}^{\prime \prime}, \\
A_{2}= & \beta(2 \alpha)^{2-n} y_{2}+\beta(2-n)\left(\epsilon x+\alpha x^{2}\right)(\gamma)^{1-n} y_{2}^{\prime \prime} \\
& +\frac{\beta(2-n)(1-n)\left(\epsilon x+\alpha x^{2}\right)(\gamma)^{-n}\left(y_{1}^{\prime \prime}\right)^{2}}{2} \\
& +\beta(2-n)(\gamma)^{1-n} y_{1} y_{1}^{\prime \prime} .
\end{aligned}
$$

Proceeding into finding $y_{1}, y_{2}, y_{3}$ via (5) we obtain

$$
\begin{aligned}
& y_{1}=\beta(\gamma)^{2-n}\left(\frac{\epsilon x^{4}}{4 !}+\frac{2 \alpha x^{5}}{5 !}\right) \text {, } \\
& y_{2}=\beta^{2}(\gamma)^{4-2 n}\left(\frac{\epsilon x^{7}}{7 !}+\frac{2 \alpha x^{8}}{8 !}\right)+\beta^{2}(\gamma)^{3-2 n}(2-n) \\
& \cdot\left(\frac{3 \epsilon^{2} x^{6}}{6 !}+\frac{20 \alpha \epsilon x^{7}}{7 !}+\frac{40 \alpha^{2} x^{8}}{8 !}\right) \text {, } \\
& y_{3}=\beta^{3}(\gamma)^{6-3 n}\left(\frac{\epsilon x^{10}}{10 !}+\frac{2 \alpha x^{11}}{11 !}\right)+\beta^{3}(\gamma)^{5-3 n}(2-n) \\
& \cdot\left(\frac{3 \epsilon^{2} x^{9}}{9 !}+\frac{20 \alpha \epsilon x^{10}}{10 !}+\frac{40 \alpha^{2} x^{11}}{11 !}\right)+\beta^{3}(\gamma)^{5-3 n}(2 \\
& -n)\left(\frac{6 \epsilon^{2} x^{9}}{9 !}+\frac{56 \alpha \epsilon x^{10}}{10 !}+\frac{112 \alpha^{2} x^{11}}{11 !}\right)+\beta^{3}(\gamma)^{4-3 n} \\
& \cdot(2-n)^{2}\left(\frac{15 \epsilon^{3} x^{8}}{8 !}+\frac{210 \alpha \epsilon^{2} x^{9}}{9 !}+\frac{1120 \epsilon \alpha^{2} x^{10}}{10 !}\right. \\
& \left.+\frac{2240 \alpha^{3} x^{11}}{11 !}\right)+\beta^{3}(\gamma)^{4-3 n}(2-n)(1-n)\left(\frac{15 \epsilon^{3} x^{8}}{8 !}\right. \\
& \left.+\frac{210 \alpha \epsilon^{2} x^{9}}{9 !}+\frac{1120 \epsilon \alpha^{2} x^{10}}{10 !}+\frac{2240 \alpha^{3} x^{11}}{11 !}\right) \\
& +\beta^{3}(\gamma)^{5-3 n}(2-n)\left(\frac{15 \epsilon^{2} x^{9}}{9 !}+\frac{112 \alpha \epsilon x^{10}}{10 !}\right. \\
& \left.+\frac{224 \alpha^{2} x^{11}}{11 !}\right)
\end{aligned}
$$


TABLE 1: Comparison between estimated values for $\gamma$ and $t_{f}$ for $n=1.5$.

\begin{tabular}{lcccc}
\hline$\epsilon$ & -0.25 & 0 & 0.5 & 0.75 \\
\hline$\gamma$ (numerical) & 0.3241 & 0.3647 & 0.2748 & 0.1720 \\
$\gamma$ (series) & 0.3243 & 0.3664 & 0.2754 & 0.1722 \\
\hline Error & 0.0002 & 0.0017 & 0.0006 & 0.0002 \\
\hline Improved $\gamma($ error $<0.0008)$ & 0.3234 & 0.3655 & 3.065 & 0.1713 \\
\hline$t_{f}$ (series) & 5.12 & 4.095 & $5.0 \%$ & 3.57 \\
Relative error in $t_{f}$ & $2.7 \%$ & $3.6 \%$ & 3.18 & 2.685 \\
\hline Improved $t_{f}$ (error $\left.<1.3 \%\right)$ & 5.235 & 4.21 & & \\
\hline
\end{tabular}

TABLE 2: Comparison between estimated values for $\gamma$ and $t_{f}$ for $n=2.5$.

\begin{tabular}{lcccc}
\hline$\epsilon$ & -0.25 & 0 & 0.5 & 0.75 \\
\hline$\gamma$ (numerical) & 0.3995 & 0.4326 & 0.3539 & 0.2534 \\
$\gamma$ (series) & 0.3945 & 0.4278 & 0.3497 & 0.2504 \\
\hline Error & -0.005 & -0.0048 & -0.0042 & -0.003 \\
\hline Improved $\gamma(\mid$ error $<0.001)$ & 0.3985 & 0.4318 & 0.3537 & 1.985 \\
\hline$t_{f}$ (series) & 3.74 & 3.026 & 6.2544 \\
Relative error in $t_{f}$ & $4.1 \%$ & $4.8 \%$ & 1.415 \\
\hline Improved $t_{f}$ (error $\left.<1.8 \%\right)$ & 3.615 & 2.901 & 1.86 & $6.7 \%$ \\
\hline
\end{tabular}

The solution must go on to $y_{4}, y_{5}, \ldots$, and so forth. We take $y \approx y_{0}+y_{1}+y_{2}+y_{3}$ as an approximate solution (which turns out to yield high accuracy relatively speaking, in approximating shear stress) and use it to estimate the values of the shear stress parameter $\gamma$ as well as the instant of time where the solution virtually reaches a constant velocity, namely, $t_{f}$, where $f^{\prime}\left(t_{f}\right)=1, f^{\prime \prime}\left(t_{f}\right)=0$. (More precisely and using the current notation $t_{f}$ is the minimum value of $x>0$ such that $y^{\prime}(x)=1$ and $y^{\prime \prime}(x)=0$ and where the last two conditions hold for all $x \geq t_{f}$.)

\section{Numerical and Series Estimates}

Using the series in the previous section as an approximation to the solution of the problem one obtains the following estimates for the initial curvature at $t=0$ (also referred to as the shear stress or vorticity parameter): namely, $\gamma=2 \alpha$ as well as $t_{f}$. We compare our results (referred to here as "series") with those obtained from numerical MATLAB integrators which are anticipated to have high accuracy (referred to here as "numerical"). We expect future works will obtain results using other numerical schemes and methods. Table 1 shows results for $n=1.5$ whereas Tables 2 and 3 show results for $n=2.5$ and $n=3$, respectively. We observe that for values of $\epsilon<1$ the estimated values of the shear stress parameter $\gamma$ are quite accurate (especially for values of $\epsilon$ closer to 1 ), as long as $n$ stays close to $n=2$, where the equation reduces to a linear equation.

The ranges we considered here for $n$ and $\epsilon$ are very practical which signifies the current results. On the other hand, while the estimation of the values of $t_{f}$ may not be as significant as that for the shear stress parameter $\gamma$ we list two observations: The needed accuracy for $t_{f}$ is not as acute/crucial as that for $\gamma$ in applications so that our estimates are considered relatively accurate. Secondly the error in $t_{f}$ is one sided; it is an underestimate for $n=1.5$ while it is an overestimate for $n=2.5$ and $n=3$. Therefore a correction can be simply done. The same observation holds true for the values of shear stress parameter $\gamma$ for $n=2.5$ and $n=3$. In fact, for $n=2.5$, adding 0.004 to each estimated value of $\gamma$ will ensure an error of less than 0.001. On the other hand, for $n=$ 3 , adding 0.0083 to each estimated value of $\gamma$ will ensure an error of less than 0.0018 . This shows that our approach proves highly accurate as the predictable error provides means to significantly improve the estimates without further/added work and effort. For $n=1.5$ the estimated values for $\gamma$ are, relatively speaking, very accurate.

It is also noted that the error increases with decreasing $\epsilon$ so that the error can further be reduced by looking for a quadratic or higher order interaction between the error and $\epsilon$ to further reduce the error but we leave the details of this analysis.

Lastly observe that for a wide range of $\epsilon \leq 0$ the error can be singled out as staying virtually constant: an observation that can help in reducing the error, with virtually no effort, to practically almost 0 once it is determined for one or two measurements for each value of interest of $n$.

\section{Conclusions}

The Adomian decomposition method was used to obtain approximate series solution for a power-law problem that has recently been studied in literature. The approximate solution was used to obtain estimates for the shear stress parameter $\gamma$ as well as the time to reach terminal point/solution $t_{f}$. It was noticed that only four terms of the infinite series were sufficient to obtain high accuracy for a certain very practical range of 
TABLE 3: Comparison between estimated values for $\gamma$ and $t_{f}$ for $n=3$.

\begin{tabular}{lcccc}
\hline$\epsilon$ & -0.25 & 0 & 0.5 & 0.75 \\
\hline$\gamma$ (numerical) & 0.4319 & 0.4624 & 0.3880 & 0.2898 \\
$\gamma$ (series) & 0.4218 & 0.4527 & 0.3795 & -0.0085 \\
\hline Error & -0.0101 & -0.0097 & 0.3896 & 0.0065 \\
\hline Improved $\gamma($ error $<0.0018)$ & 0.4301 & 0.4610 & 1.785 & 0.2916 \\
\hline$t_{f}$ (series) & 3.45 & 2.805 & $9.9 \%$ & 1.22 \\
Relative error in $t_{f}$ & $7.0 \%$ & $8.0 \%$ & 1.735 & $10.7 \%$ \\
\hline Improved $t_{f}$ (error $\left.<4.1 \%\right)$ & 3.4 & 2.755 & 1.17 \\
\hline
\end{tabular}

the power-law index $n$. A crucial observation in this context is that the error in the measured parameters is predictable which makes it possible to significantly reduce the error with virtually no effort. This is a peculiar phenomenon which we conjecture to apply to many other power-law problems of non-Newtonian fluids.

\section{Competing Interests}

The authors declare that there are no competing interests regarding the publication of this paper.

\section{References}

[1] H. Blasius, "Grenzschichten in Flüssigkeiten mit kleiner Reibung," Zeitschrift für Angewandte Mathematik und Physik, vol. 56, pp. 1-37, 1908.

[2] M. Guedda and Z. Hammouch, "Similarity flow solutions of a non-Newtonian power-law fluid," International Journal of Nonlinear Science, vol. 6, no. 3, pp. 255-264, 2008.

[3] M. Guedda, "Boundary-layer equations for a power-law sheardriven flow over a plane surface of non-Newtonian fluids," Acta Mechanica, vol. 202, no. 1-4, pp. 205-211, 2009.

[4] A. Nachman and S. Taliaferro, "Mass transfer into boundary layers for power law fluids," Proceedings of the Royal Society of London Series A: Mathematical and Physical Sciences, vol. 365, no. 1722, pp. 313-326, 1979.

[5] L. Zheng, X. Zhang, and J. He, "Existence and estimate of positive solutions to a nonlinear singular boundary value problem in the theory of dilatant non-Newtonian fluids," Mathematical and Computer Modelling, vol. 45, no. 3-4, pp. 387-393, 2007.

[6] T. G. Howell, D. R. Jeng, and K. J. De Witt, "Momentum and heat transfer on a continuous moving surface in a power law fluid," International Journal of Heat and Mass Transfer, vol. 40, no. 8, pp. 1853-1861, 1997.

[7] X.-H. Chen, L.-C. Zheng, and X.-X. Zhang, "MHD boundary layer flow of a non-newtonian fluid on a moving surface with a power-law velocity," Chinese Physics Letters, vol. 24, no. 7, pp. 1989-1991, 2007.

[8] X. Su, L. Zheng, and J. Feng, "Approximate analytical solutions and approximate value of skin friction coefficient for boundary layer of power law fluids," Applied Mathematics and Mechanics, vol. 29, no. 9, pp. 1215-1220, 2008.

[9] G. Bognár, "Similarity solution of a boundary layer flows for non-Newtonian fluids," International Journal of Nonlinear Sciences and Numerical Simulation, vol. 10, no. 11-12, pp. 15551566, 2009.
[10] M. C. Ece and E. Büyük, "Similarity solutions for free convection to power-law fluids from a heated vertical plate," Applied Mathematics Letters, vol. 15, no. 1, pp. 1-5, 2002.

[11] S.-J. Liao, "A challenging nonlinear problem for numerical techniques," Journal of Computational and Applied Mathematics, vol. 181, no. 2, pp. 467-472, 2005.

[12] D. M. Wei and S. Al-Ashhab, "Similarity solutions for nonNewtonian power-law fluid flow," Applied Mathematics and Mechanics, vol. 35, no. 9, pp. 1155-1166, 2014.

[13] H. Schlichting, Boundary Layer Theory, McGraw-Hill Press, New York, NY, USA, 1979.

[14] G. Bohme, Non-Newtonian Fluid Mechanics, North-Holland Series in Applied Mathematics and Mechanics, Elsevier Science, Amsterdam, The Netherlands, 1987.

[15] S. Al-Ashhab, "A curvature-unified equation for a nonNewtonian power-law fluid flow," International Journal of Advances in Applied Mathematics and Mechanics, vol. 2, no. 3, pp. 72-77, 2015. 


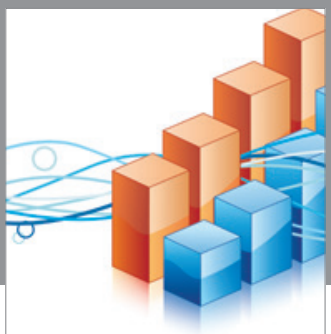

Advances in

Operations Research

vatem alat4

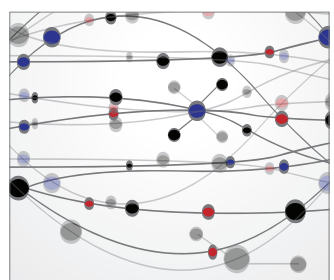

\section{The Scientific} World Journal
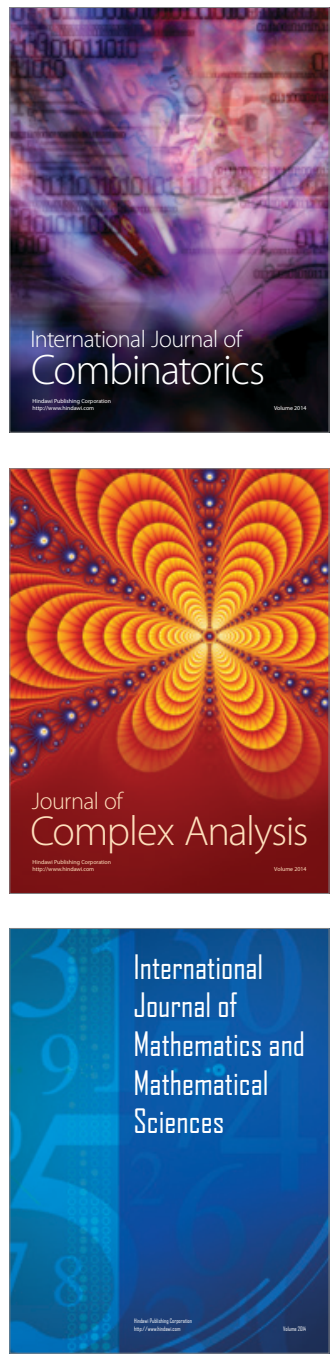
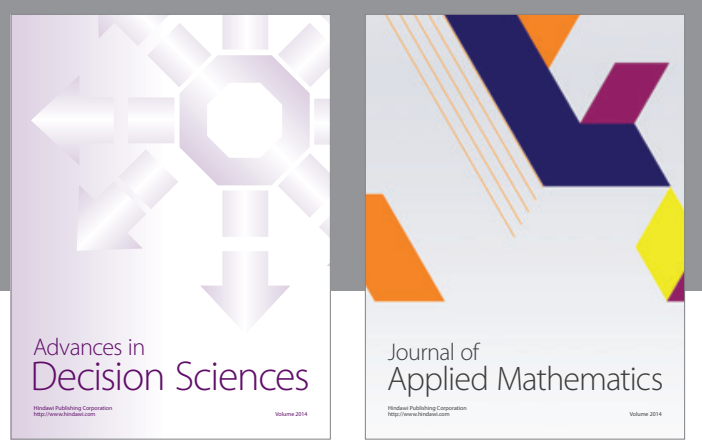

Algebra

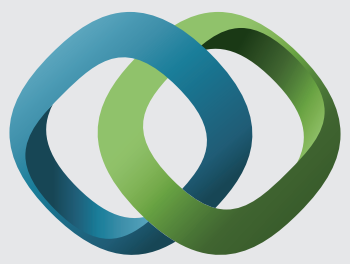

\section{Hindawi}

Submit your manuscripts at

http://www.hindawi.com
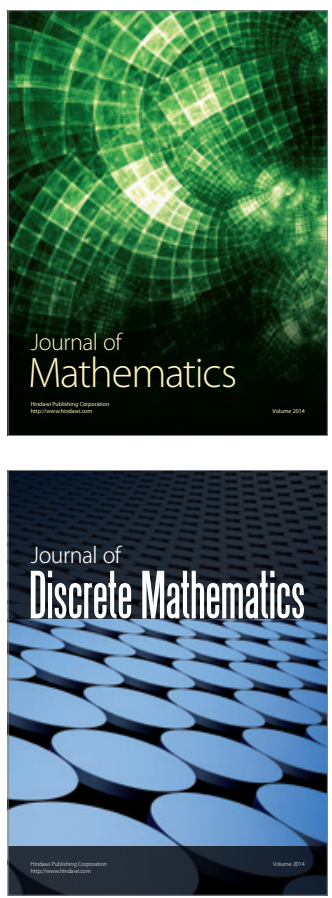

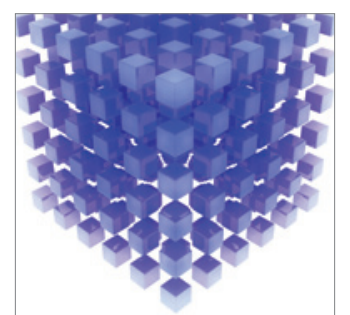

Mathematical Problems in Engineering
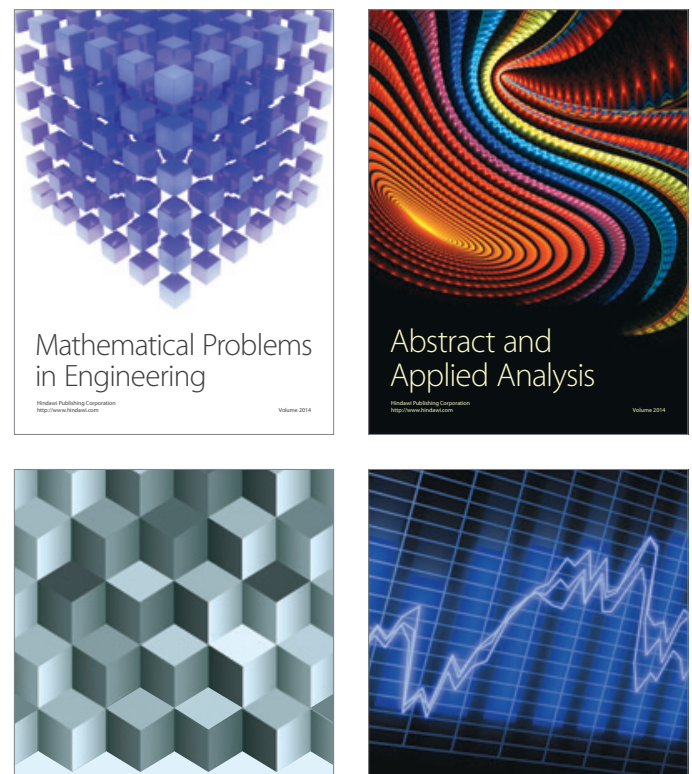

Journal of

Function Spaces

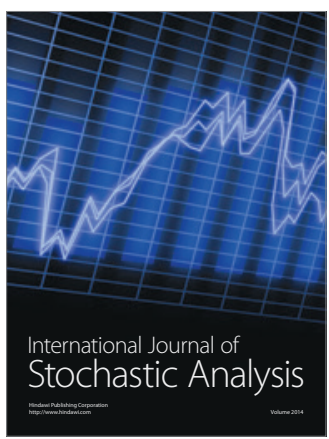

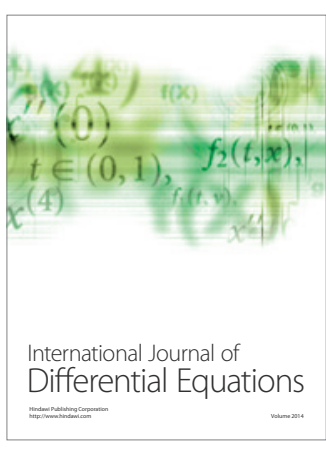
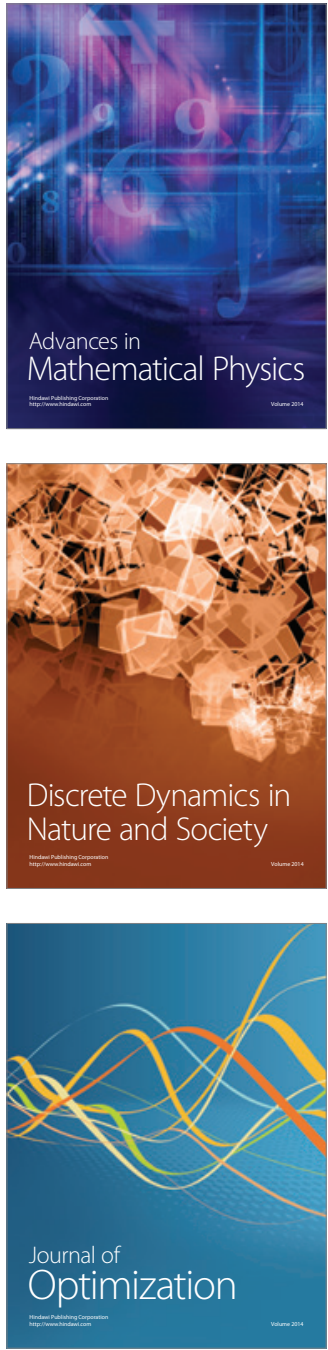